\title{
New data analysis to evaluate defects in composite materials using microwaves thermography
}

\author{
by U. Galietti*, D. Palumbo*, G. Calia* and F. Ancona* \\ *Dipartimento di Ingegneria Meccanica e Gestionale (DIMEG), Politecnico di Bari, Italy, galietti@oliba.it, \\ d.palumbo@poliba.it, caliagianfranco@poliba.it, f.ancona@studenti.poliba.it
}

\begin{abstract}
In this work microwave sources were used as heating sources to evaluate the damages areas in composite materials using thermographic techniques. In particular, lock-in thermography and microwave lock-in thermography tests were carried out on CFRP damaged specimens. A new data analysis was developed to processing the thermographic data obtained by microwave tests and various algorithm were used to processing lock-in thermography tests. Data obtained were compared with non destructive tests performed with other techniques such as $\mathrm{x}$-ray. A numerical model was developed for microwave heating simulation.
\end{abstract}

\section{Introduction}

Nowadays composite materials are used in many application fields such as aeronautical industry and non destructive techniques represents a necessary tool to diagnostic damages in these materials. In particular, well established techniques such as ultrasound and x-ray and traditional thermographic techniques such as pulsed and lockin thermography are used to detect the largest variety of defects in a short time. Thermographic techniques are full-field techniques with a relatively simple set-up and allow a rapid investigation of materials $[1,2,3,4,5]$. Interesting results were obtained using the TSA (Thermoelastic Stress Analysis) on composite materials. In this case the cracks are detected by means a dynamic load applied on materials $[6,7]$.

These techniques have some limitations in detecting of very small defects placed at depths greater than $10 \mathrm{~mm}$ and many problems can occur in the case of automatic detection of defects.

In this work has been used microwave as heating source for non destructive evaluation of composite material. The aim of the work is detect the defects that traditional thermographic techniques are not able to detect.

Microwaves are used to heating water inside materials; the presence of small defects e.g., defects due to impact damage, can cause the penetration of water inside the composite moisture. In particular environmental conditions, water inside materials may freeze with the possible size increase of defects [8]. This means that detecting the moisture inside the composite components, is important to predict irreversible damage of the components.

The tests have been carried out on composite material specimens, in particular preliminary tests have been carried out to optimize the experimental set-up on CFRP specimens manufactured with resin film infusion technique. To quantitative analysis of defects have been used impact damaged CFRP specimens.

The specimens have been heated using a commercial microwave oven suitably modified. In particular, microwaves sensors have been used to measure leaks from microwave oven during the test.

A numerical model was developed to simulate the microwave heating and to compare the results with experimental analysis. Numerical results are a valuable tool to predict and understand the components behavior in terms of the thermal, mechanical and electrical not homogeneous characteristics of composite materials. The data obtained with experimental tests were used to set the numerical analysis as close as possible to real model. The software used for the simulation was COMSOL Multiphysics.

\section{Experimental set-up}

Lock-in thermography tests have been carried out on specimens in composite material, in particular, CFRP specimen manufactured with RFI technique (Resine Film Infusion) and a sandwich specimen in sintattic foam, "Henkel Syncore 9872.1, K40".

The RFI specimen was subjected to impact test with $30 \mathrm{~J}$ impact energy. After the impact the specimen was tested to measure the residual strength, according to standard ASTM, causing a break on the right side as shown in figure 1.

The sandwich specimen was obtained with foam layers assembled CFRP with preimpregnated layers. For high impact energy $(E=70 \mathrm{~J})$ the laminate layers in carbon fiber undergo matrix breaks also in sub superficial layers and delaminations can create also between layers. The specimen used for tests has following dimension: width $76 \mathrm{~mm}$, length $76 \mathrm{~mm}$ and thickness $8 \mathrm{~mm}$. 

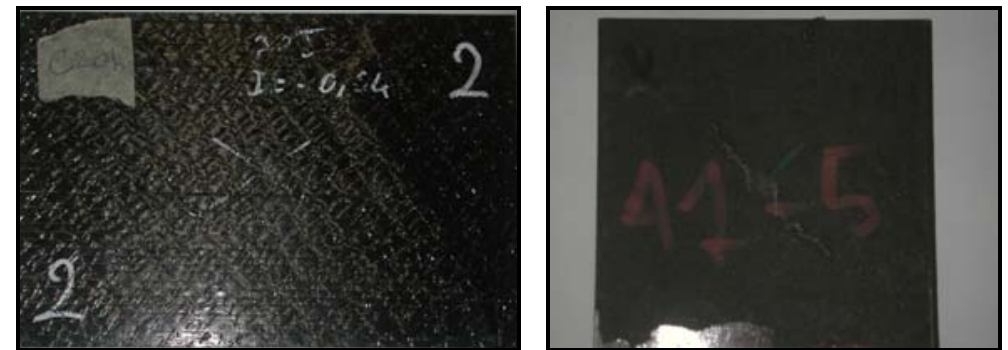

Fig.1. RFI (left) specimen and sandwich specimen (right)

Thermographic tests have been carried out by means commercial domestic microwaves oven. (power $750 \mathrm{~W}$, frequency $2450 \mathrm{~Hz}$, capacity $20 \mathrm{l}$ ). The microwave oven was modified to improve the tests performance, in particular the internal lamp was removed because during the test it overheated e became a heat source. After preliminary tests and when only cooling phase was recorded, the oven protection glass was removed in order to recording the temperature trend of specimen during heating phase. A copper grate permits a totally reflection of microwaves coming to the inside of the oven and allows the protection of instrumentation, such as the thermocamera, placed in front of the oven. The domestic microwave oven produce $2,45 \mathrm{Ghz}$ frequency waves suitable to excite water molecule and at length of about $12 \mathrm{~cm}$, much more greater than the copper grate knit.

During preliminary tests various measurement have been carried out to evaluate possible microwaves leakage using electromagnetic sensors placed in various positions close to oven figure 2.

\section{8,11}

\begin{tabular}{l|lll|l|}
\hline 0,04 & 0,67 & 0,49 & \\
0.45 & 0,21 & 04 & 0,1 & \\
0,87 & 0,25 & 0,76 & \\
\hline
\end{tabular}

Fig.2. Electromagnetic waves evaluated in various point on the front door of the microwaves oven

The thermography data have been acquired via two different thermocameras, in particular for the early tests have been used a 160×120 microbolometer detector ThermoVision A20 M, which work in the long wave of the infrared spectrum between $7 \mu \mathrm{m}$ and $14 \mu \mathrm{m}$ (NETD $<55 \mathrm{mK}$ ). These tests have been used to identify the possible optimal set-up. After, the tests have been performed by differential IR camera DeltaTherm 1560 (DT) made by StressPhotonics (USA) with thermal sensitivity (NETD) $<18 \mathrm{mK}$ and based on a InSb photonic detector with $320 \times 256$ pixels. With both thermocameras were acquired thermographic sequences at $50 \mathrm{~Hz}$ frequency with Flir A20 and at $100 \mathrm{~Hz}$ frequency with DeltaTherm 1560.

In order to obtain the optimal set-up was taking in account the following parameters: specimen position in oven, presence of a water container in oven, position of a water container in oven, heating time and position of thermocamera.

The specimen was positioned in the centre of oven in order to receive the great part of microwave coming from magnetron and to obtain a short heating time. In fact different temperatures were measured changing the position of the specimen in the microwaves oven.

The heating time has been chosen considering that, elevated heating time can cause high temperatures that can damage the composite material, while low heating time doesn't allow to obtain sufficient thermal contrast to detect defects. In this case a 5 second heating time has been used for the tests without the presence of water container in the oven.

The thermocamera has been positioned at $15 \mathrm{~cm}$ from grate to permits to focus and to frame the whole specimen and to avoid damage due to possible microwaves leakage from oven figure 3 . 


\section{Microwave Oven}

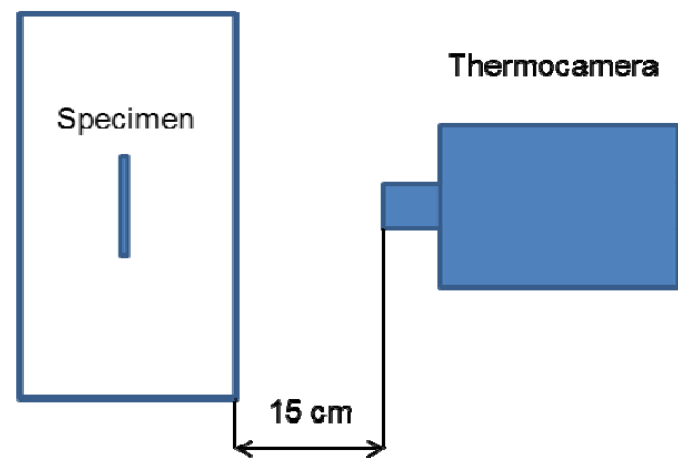

Fig.3. Experimental set-up used for microwaves thermography

In the lock-in test was used a halogen lamp with power $500 \mathrm{~W}$, while signal processing was made with DeltaTerm software. The table 1 show the parameters used for lock-in thermography tests.

Table 1. Lock-in thermography tests on sandwich specimen

\begin{tabular}{|c|c|c|c|c|}
\hline $\mathrm{N}^{\circ}$ test & Period $[\mathrm{s}]$ & Frequency $[\mathrm{Hz}]$ & $\begin{array}{c}\text { Frame } \\
\text { acquired }\end{array}$ & $\begin{array}{c}\text { Thermocamera acquisition } \\
\text { speed }[\mathrm{Hz}]\end{array}$ \\
\hline 1 & 1 & 1 & 20 & 5 \\
\hline 2 & 3 & 0,33333 & 60 & 5 \\
\hline 3 & 5 & 0,2 & 90 & 5 \\
\hline 4 & 10 & 0,1 & 175 & 5 \\
\hline 5 & 20 & 0,05 & 350 & 2 \\
\hline 6 & 60 & 0,01667 & 380 & 2 \\
\hline 7 & 90 & 0,01111 & 630 & 2 \\
\hline 8 & 120 & 0,00833 & 840 & \\
\hline
\end{tabular}

The $x$-ray image (figure 4) show the presence of delaminate zone near the impact zone in the superficial composite layers. In the impact zone there are delamination in deep areas of the specimen that involve the foam also.

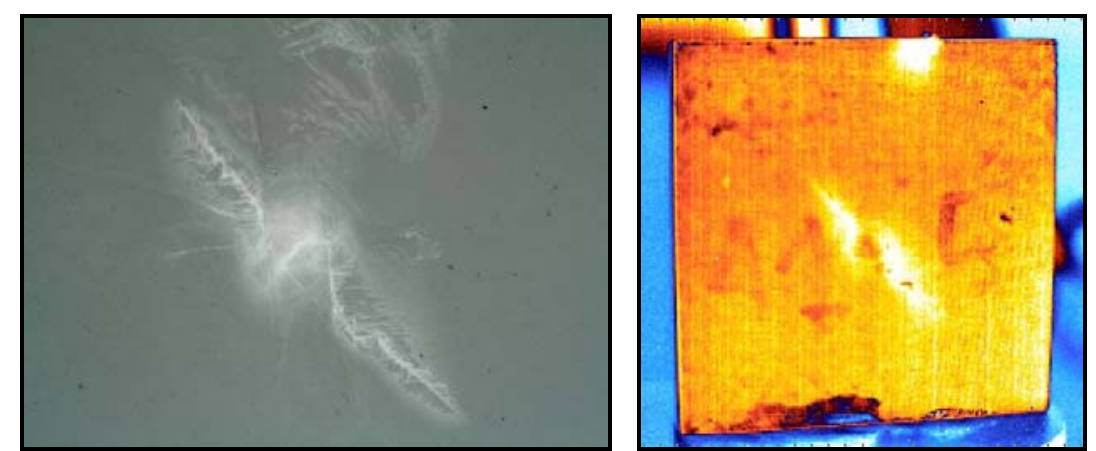

Fig. 4. X-ray digital image (left) and lock-in thermography phase image ( $f=0.1 \mathrm{~Hz})$ (right)

The lock-in thermography phase image in figure 4, confirms the presence of delaminations in the centre of the specimen and provides less information on impact zone in comparison to x-ray tests. Moreover with lock-in thermography technique has been detected another delamination on the edge of the specimen.

\section{Numerical model}

A microwave oven cavity, with dimensions $267 \times 270 \times 188$ [mm] was used to simulate the electromagnetic field distribution. Figure 5 shows the configuration used for numerical analysis with the specime placed at hthe centre on the oven. The magnetron (output power of 700 [W]) radiates with a frequency of $2.45 \mathrm{GHz}$. The port $(50 \times 78 \times 18$ [mm]) has been excited with the fundamental mode $\mathrm{TE}_{10}$ (Transverse Electric) $[9,10]$. The $\mathrm{TE}_{10}$ mode was used to generate the microwave through the rectangular waveguide. In fact in this case the frequency is determined by: 


$$
\left(f_{m n}\right)=\frac{c}{2} \sqrt{\left(\frac{m}{y}\right)^{2}+\left(\frac{n}{z}\right)^{2}}
$$

where $m$ and $n$ are the mode numbers, $c$ denotes the speed of light. The dimensions of the rectangular cross section is $y=78[\mathrm{~mm}]$ and $z=18[\mathrm{~mm}]$ so the TE10 mode provides the followed range of frequencies $1.92 \mathrm{GHz}(\mathrm{m}=1)$ and $3.84 \mathrm{GHz}(\mathrm{m}=2)$.

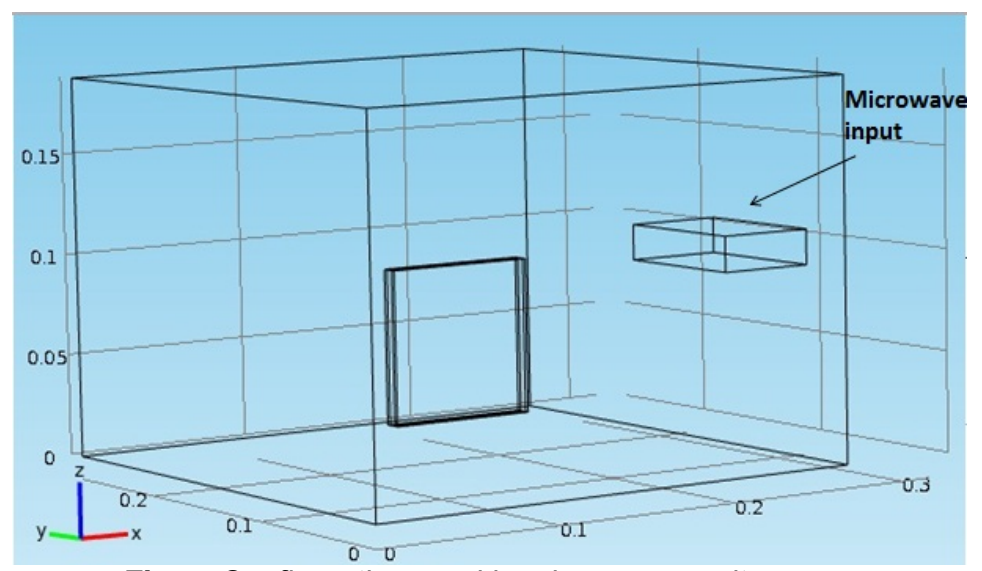

Fig. 5. Configuration used in microwave cavity oven

The mesh used is a tetrahedral type for both geometries that provides an optimal resolution to this type of 3D simulation. A different element size was used for the specimen and oven, in particular for the oven was used an element size of $7.5[\mathrm{~mm}]$ while for the specimen was used an element size of $1.5[\mathrm{~mm}]$ (figure 6).
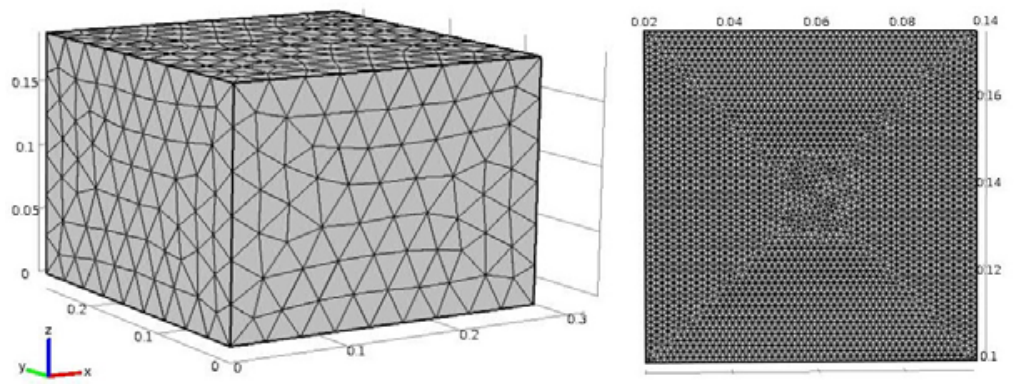

Fig. 6. Mesh size for the oven (left) and the specimen (right)

To simulate the electric field, was used the COMSOL Multiphysics software. The microwave radiation coming from a rectangular port, as shown in figure 5 . The electric field vector is calculated using:

$$
\nabla \times\left(\mu^{-1} \nabla \times E\right)-\omega^{2} \varepsilon_{c}=0
$$

where $\sigma$ is the electrical conductivity, $\varepsilon_{c}$ is the complex permittivity and $\mu$ the magnetic permeability [10]. The absorbed power density of the material can be expressed by:

$$
P=\frac{1}{2}\left[\left(\sigma+\omega \varepsilon_{c}\right) E^{2}\right]
$$

To properly set the specimen CFRP characteristics material (thermal and electric) is necessary compare the experimental results with numerical results. In particular, comparing the heating and cooling curves of material is possible to define the correct values of composite characteristics material such as, thermal and electrical conductivity, relative permittivity and permeability.

The presence of the defect was simulated with a lack of material inside the specimen containing humid air in order to simulate the presence of water. Several tests were carried out varying the volume size and the depth of the defect. 


\section{Results}

\subsection{Experimental results}

The aim of this work is use an innovative heating technique using microwaves to detect deep defect in composite materials with thermography.

The substances have an electric dipole and tend to align their angular momentum to electric field produced by microwaves. This alignment causes an elongation of dipolar atoms and this movement, causes friction between atoms with positive charge and atoms with negative charge, creates heating. The water is a dipolar substance hence, the objects that contain water can be heated using microwaves. In the damaged zones of material there is a moisture accumulation, therefore the microwaves can represent a valid tool to investigation of wet zones [8].

The presence of a grid between specimen and thermocamera tends to filter the infrared radiation directed towards the thermocamera. In order to evaluate the effective temperature of the specimen has been necessary to compute the trasmittance value $\mathrm{T}$ of the grid in the temperature range of test. The $\mathrm{T}$ value has been calculated using a reference blackbody (Hart Scientific 4180 Fluke) in order to obtain a trasmittance curve which allows to obtain the specimen real superficial temperature in function of the apparent temperature measured.

Monitoring the heating and the cooling curve of specimen, a different behaviour between undamaged zone and damaged zone of the specimen was observed. In particular, in undamaged zone the heating curves present an initial linear trend. The slope of the linear curve increases with amount of microwaves received by specimen.

After the heating phase, the temperature slight decreases, due to heat diffusion phenomena probably towards deeper undamaged zone, which are less heated by microwaves. The temperature tends to stabilize towards a constant value in the last phase of cooling (figure 7).
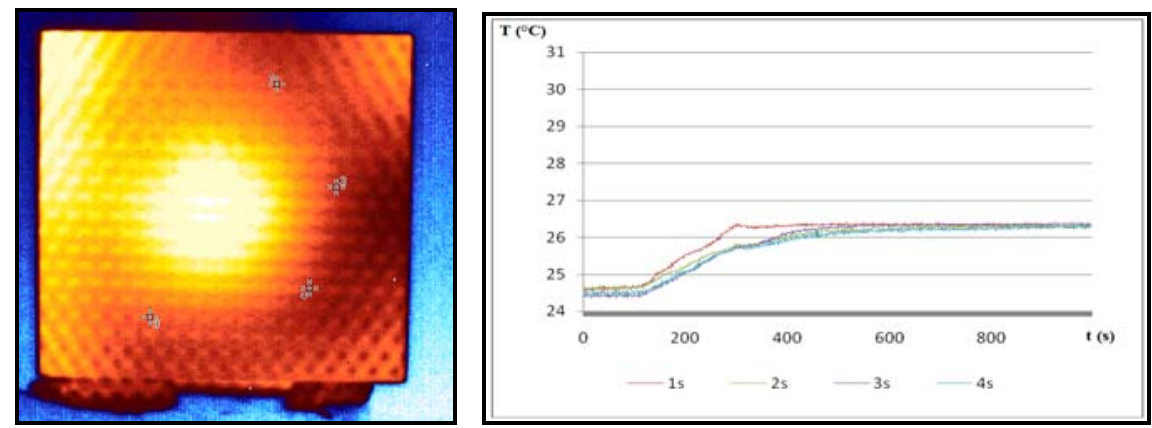

Fig. 7. Temperature-time graph for 4 point on undamaged areas
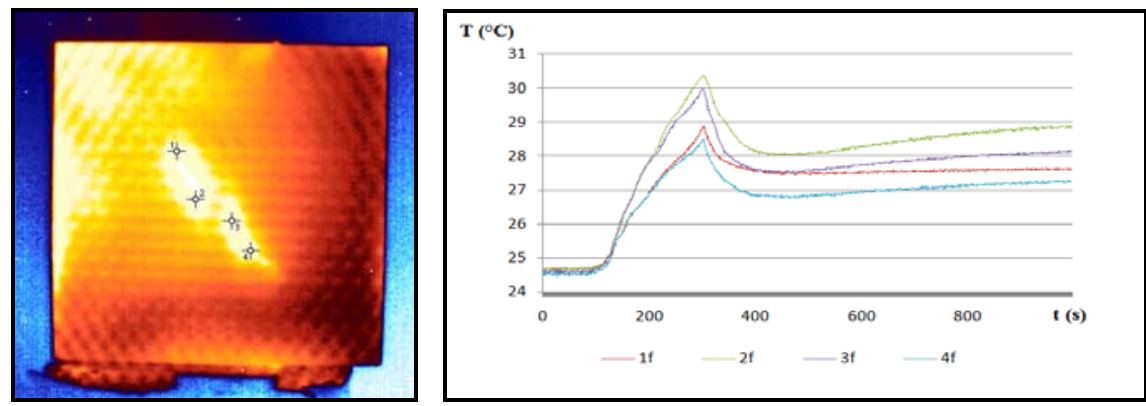

Fig. 8. Temperature-time graph for 4 point on delaminations areas
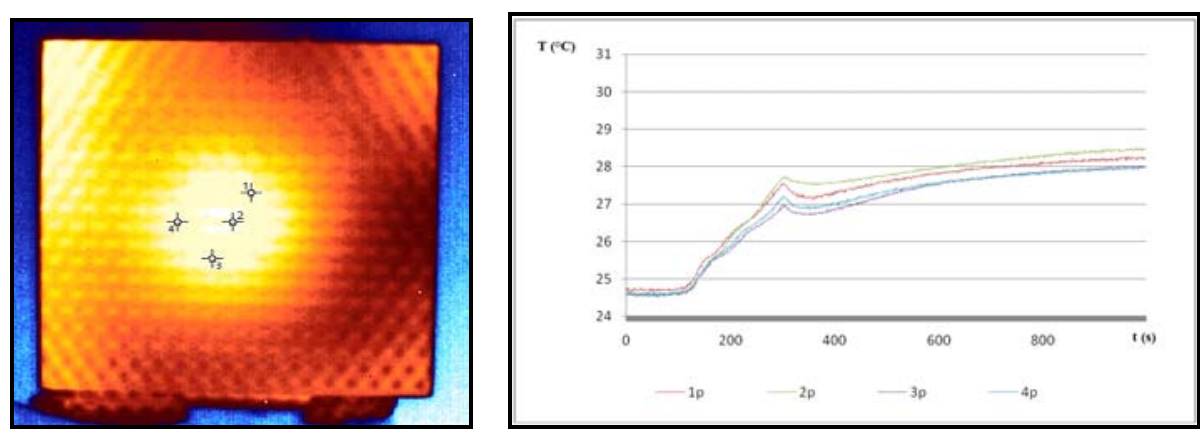

Fig. 9. Temperature-time graph for 4 point on impact areas 
On specimen surface are evident two delaminations in the central zone on the side of impact zone and there are cracks in the carbon fibers along the fibers direction.

In the delamination zones, the heating of the material occurs in a very sudden manner with a curve heating slope much higher respect to undamaged zones.

At the end of the heating there is a very rapidly cooling phase and a subsequent temperature increment. In fact in the central damaged zone of the specimen, there are sub superficial defects caused by impact test and then there is a temperature increase caused by the heat coming to damaged zones placed in more deep thickness of the specimen (figure 8). These defects are placed in the interface between carbon fibers laminate and Syncore resin. In this case the heating curve is less linear than undamaged and damaged superficial zones. At the end of the heating there is a short cooling followed by a temperature increase up to achieve a constant value figure 9.

An innovative algorithm was developed to detect in automatic mode, different defects typology placed in different deep of material.

The algorithm is based on hypothesis then the most points of the specimen are undamaged. The data analysis is based on the average behaviour of all points of specimen over time. The defected points have a different behaviour over time respect to the average behaviour previously evaluated. In particular, the damaged zones are identified considering two criteria: the maximum achieved temperature and the slopes of the heating and cooling phase during the test. Figure 10 show a generic curve of one point obtained during the test characterized by a first phase (phase 1) due to heating of the material until to the maximum value of temperature (Tmax), by a second phase (phase 2) subsequent to the switch off of the magnetron of the oven and by a third phase (phase 3), different point by point, that provides important information on the defects typology.

The algorithm developed in Matlab®, acquires a thermografic sequence, calibrates the thermal data in order to obtain the temperature values considering the trasmittance coefficient of the grid, evaluates for each point of specimen, the temperature-time curve, the maximum temperature and the curves slope of each phase.

Figure 10 shows the slope matrix evaluated during the heating phase (phase 1). The superficial damaged zones are visible in the central part and on top of the specimen. The superficial damage points have a very steep slope heating curve respect to undamaged points.

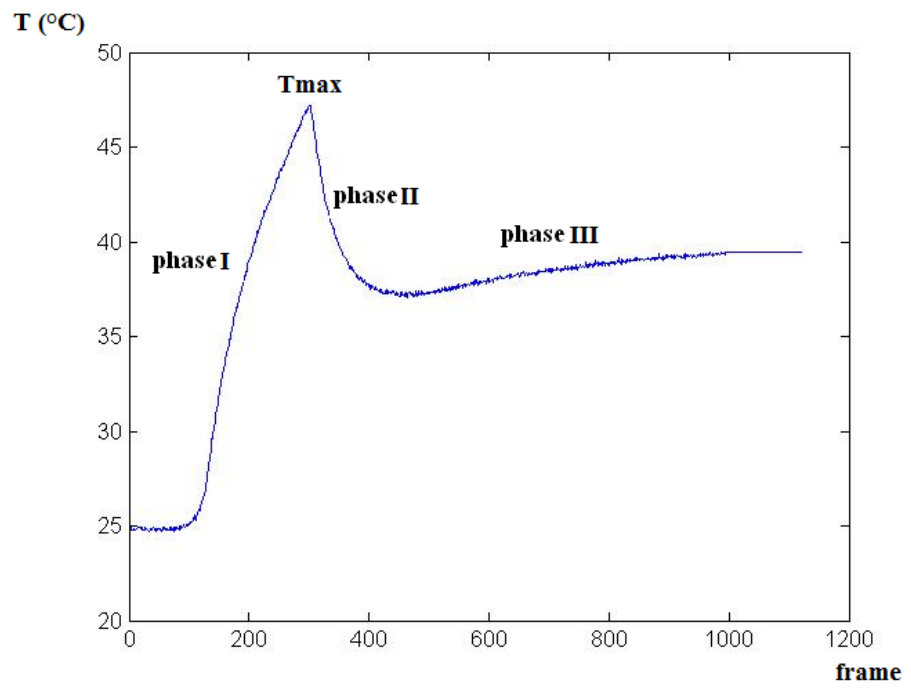

Fig. 10. Temperature trend over time and 3 phase obtained during the tests

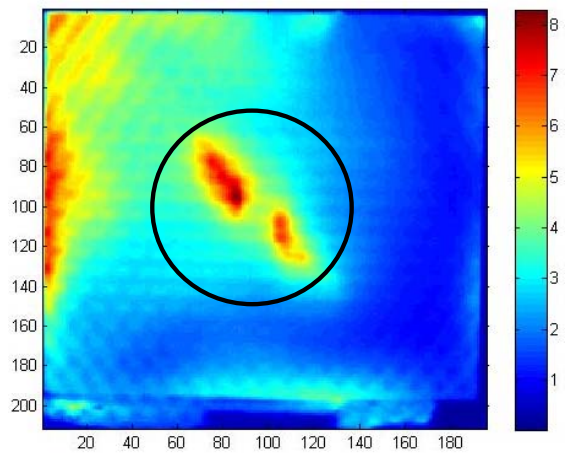

Fig. 11. Slope matrix evaluated during the heating phase (phase 1) 


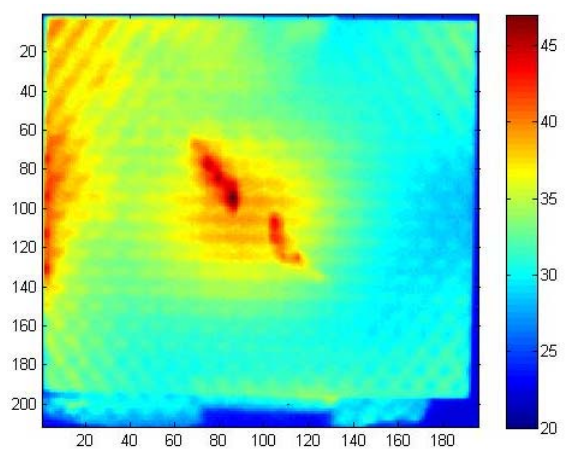

Fig. 12. Matrix of maximum temperature

The figure 12 shows the matrix of maximum temperature for each point. In this case is possible have information also on the presence of deeper defects. In particular in this case is present in the central zone of the specimen a circular damaged zone due probably to break among core and CFRP laminate. The images show also an anomalous heating on the edge of the specimen due to non-uniform heating produced by microwaves oven.

A comparison with $\mathrm{x}$-ray technique and lock-in thermographic technique has been carried out to validate the new procedure adopted. In order to obtain a quantitative data analysis has been considered an equivalent defect area determined by a circular area with diameter equal to maximum dimension of the defect area obtained by NDT tests. Figure 10 shows the circular area adopted to quantitative analysis (black circle) [11]. The measurement of this area has been made applying maximum contrast method [4] on thermographic image, knowing $\mathrm{mm} /$ pixel ratio. The evaluation on $x$-ray images have been carried out using digital images and digital ruler.

The table 2 summarize the obtained results. The value of the equivalent area are comparable in the case of $x-$ ray and microwaves thermography techniques while with lock-in thermography, the measurement of the equivalent area is overestimate respect to the x-ray. The quantitative data analysis confirms the possibility to use the microwaves as thermal source to non-destructive control of composite materials.

Table 2. Lock-in thermography tests on sandwich specimen

\begin{tabular}{|c|c|}
\hline Technique & Equivalent Area $\left[\mathbf{m m}^{2}\right]$ \\
\hline Microwave thermography & 1693 \\
\hline Lock-in thermography & 9431 \\
\hline X-ray & 1385 \\
\hline
\end{tabular}

\subsection{Numerical results}

The numerical simulation and the experimental tests are certainly a very useful tool in the description of the physical phenomena.

To determine the thermal and electrical parameters of the material have been carried out numerical preliminary tests varying the value of parameters and evaluating the temperature trend. The evaluated parameters were: electrical conductivity $(\sigma)$, thermal conductivity $(k)$ and the heat capacity at constant pressure $(\mathrm{Cp})$. All parameters present a linear decreasing trend of the average temperature evaluated on the surface of the specimen as shown in figure 13.

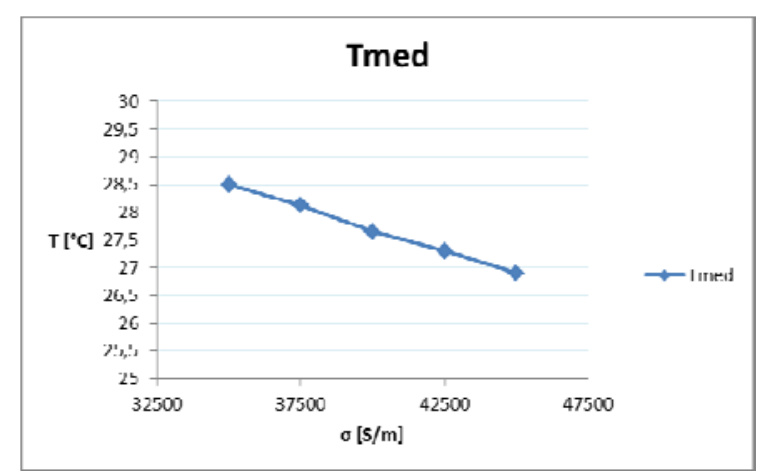

Fig. 13. Temperature vs. electrical conductivity

In particular the electrical and the thermal conductivity affect the temperature values during the heating while the thermal conductivity and the heat capacity at constant pressure affect the temperature values during the cooling.

In the table 3 are shown the results obtained comparing the numerical and experimental analysis. 
Table 3. Material parameters

\begin{tabular}{|c|c|}
\hline \multicolumn{2}{|l|}{ Thermal parameters } \\
\hline Thermal conductivity $(\mathrm{k})$ & $4\left[\mathrm{~W} / \mathrm{m}^{*} \mathrm{~K}\right]$ \\
\hline Constant-pressure heat capacity $(\mathrm{Cp})$ & $600\left[\mathrm{~J} / \mathrm{Kg}^{*} \mathrm{~K}\right]$ \\
\hline \multicolumn{2}{|l|}{ Electrical parameters } \\
\hline Electrical conductivity $(\sigma)$ & $3,5^{*} 10^{4}[\mathrm{~S} / \mathrm{m}]$ \\
\hline
\end{tabular}

The undamaged points of the specimen do not exhibit abnormal heating, in fact, the heating curve has a linear trend, the curve slope depends primarily by the microwave power and in particular by the electric distribution field.

The specimens presents two different zones: a central zone characterized by an higher value of the electric field, which causes an abnormal heating, with a great slope of the temperature curve over time and the edges characterized by high temperature due to concentration of the electric field.

In the cooling phase the temperature present two different trends:

1. in the central area there is a slight cooling, probably due to the diffusion phenomena towards the deep zones of the specimen at lower temperature. The temperature tends to stabilize over time;

2. in the area at a lower temperatures a slight increase of temperature is due to heat conduction from high temperature areas to lower temperature areas.

Figure 14 shows the analyzed points and the heating-cooling curves: there is a linear trend of the temperature curve during the heating phase, in both points.
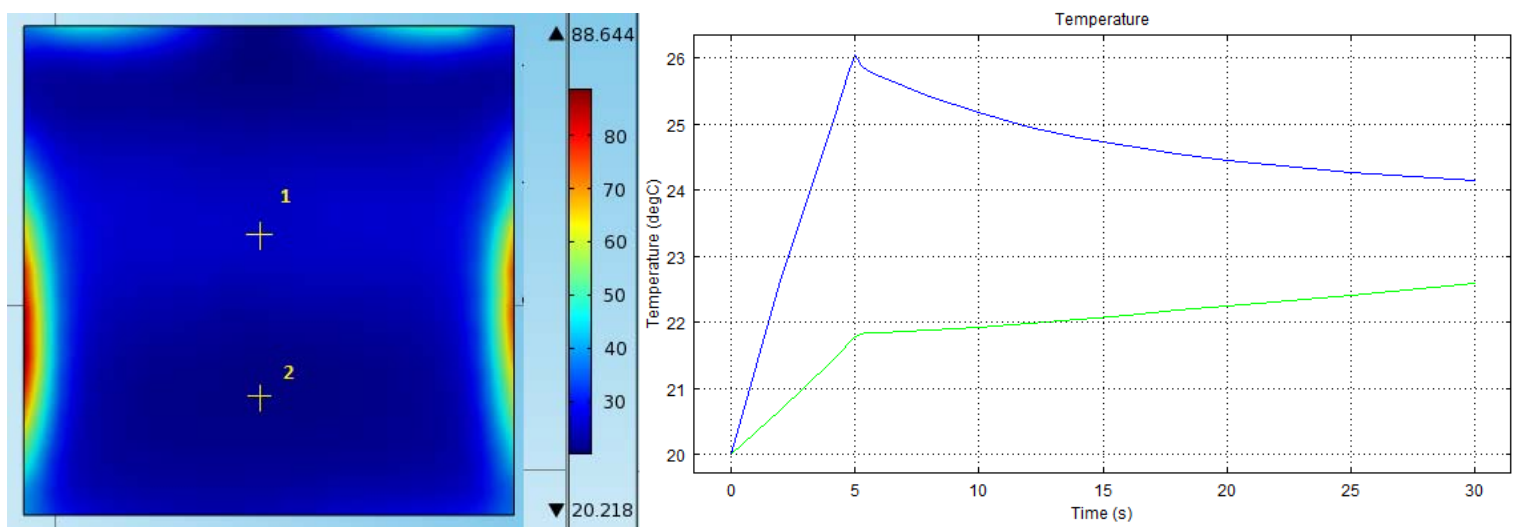

Fig. 14. Undamaged specimen (left) with heating-cooling temperature curves of two points (right)

Figure 15 shows the damaged specimen with the presence of the defect in the central zone. The surface themperature in defect zone at the end of heating gradually decreases, increasing the depth and decreasing the thickness of the defect.

Figures 15, 16 and 17 show the heating-cooling temperature curve obtained changing the defect dimension. As just viewed in the experimental tests, the defect can be detected considering:

- $\quad$ the thermal contrast generated during the test (Tmax);

- the trend of the heating-cooling curves in damaged and undamaged areas.
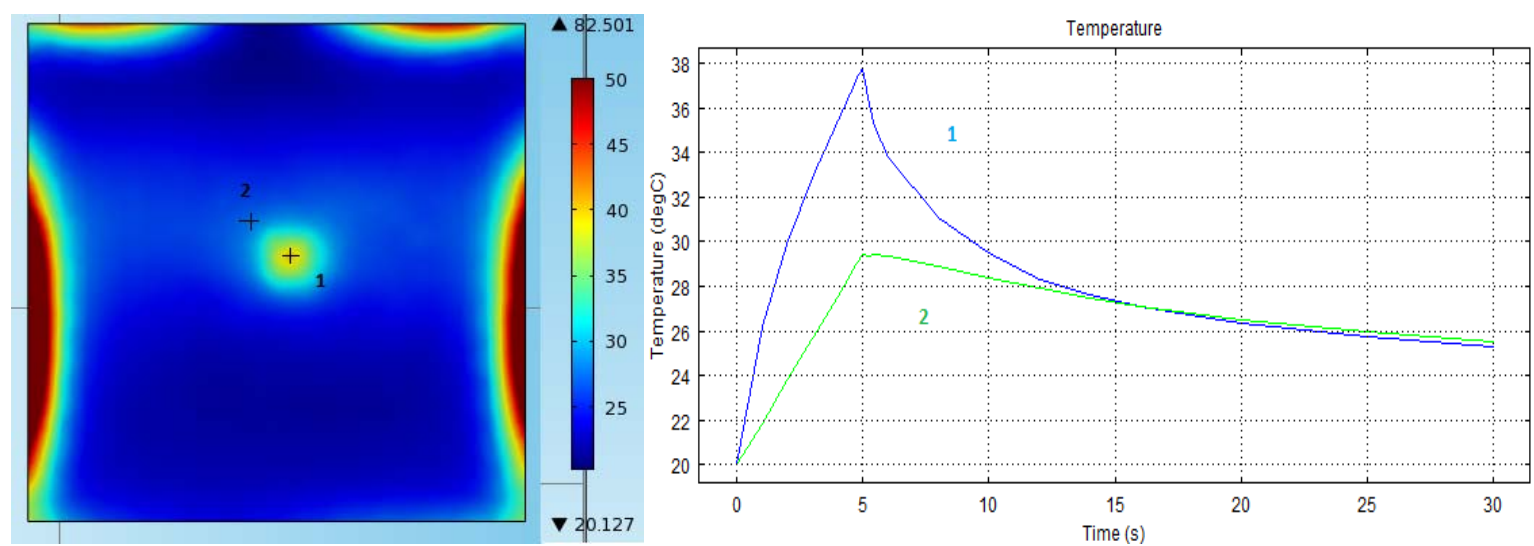

Fig. 15. Defect $6 \times 6 \times 1[\mathrm{~mm}]$ placed at depth of $0,3[\mathrm{~mm}]$ (left) with heating-cooling temperature curves of two points (right) 
All figures show a non-linear trend of temperature heating curve of damaged zones (curve 1) respect to linear trend obtained for undamaged zones (curve 2).

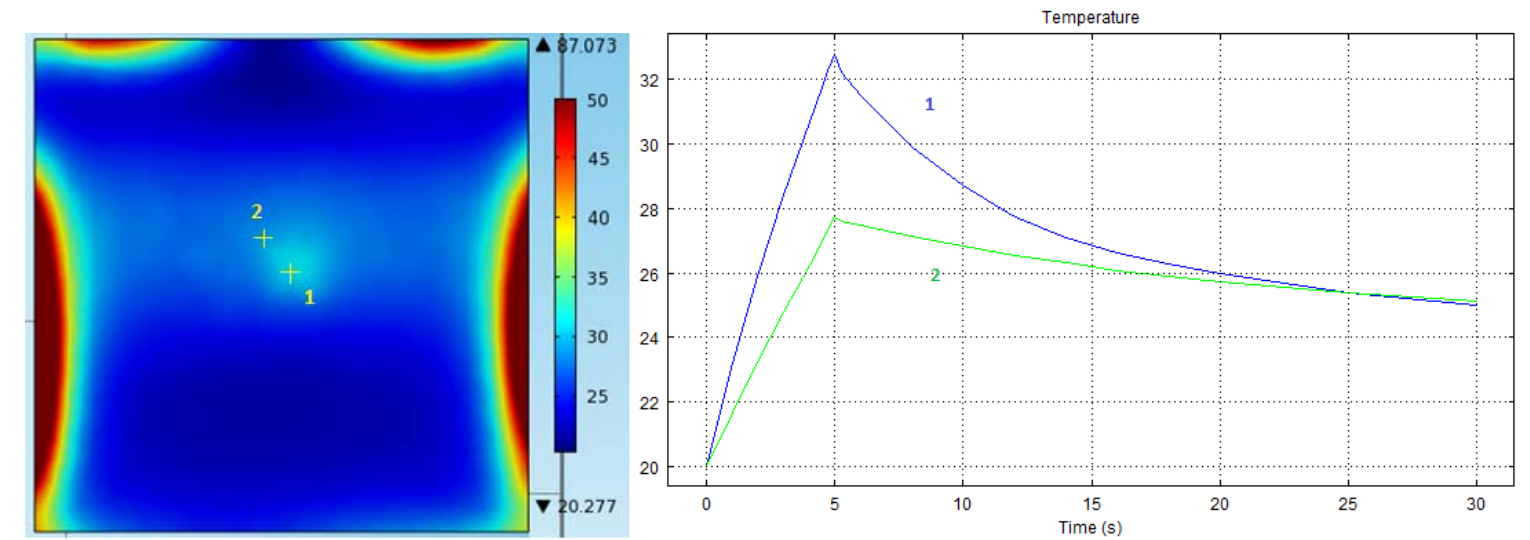

Fig. 16. Defect $6 \times 6 \times 1$ [mm] placed at depth of 1,3 [mm] (left) with heating-cooling temperatur curves (right)

The thermal contrast evaluated among damaged and undamaged zones decrease when defect depth increase and when the defect size decrease. Figures 15 and 16 show the same defect placed to different depth $(0,3$ and 1,3 $[\mathrm{mm}]$ ); a temperature variation of $5\left[{ }^{\circ} \mathrm{C}\right]$ was obtained between the maximum temperature reached on the surface of the specimen. Figures 16 and 17 show different size defects placed to the same depth $(1,3[\mathrm{~mm}])$; a temperature variation of $1,5\left[{ }^{\circ} \mathrm{C}\right]$ was obtained between the maximum temperature reached on the surface of the specimen.
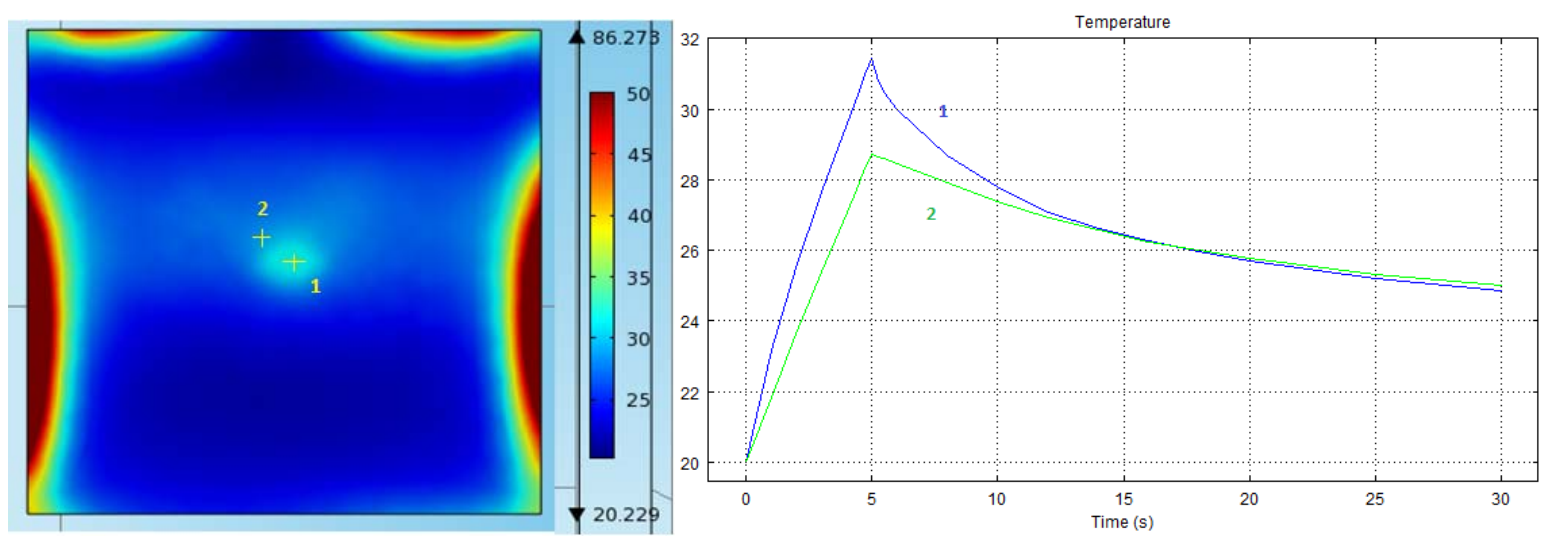

Fig. 17. Defect $2 \times 2 \times 0,5$ [mm] placed at depth of $1,3[\mathrm{~mm}]$ (left) with heating-cooling temperature curves (right)

As shown in figure 18 is difficult detect the very little defects (about $1 \times 1 \mathrm{~mm}^{\wedge} 2$ ), characterized by thermal contrast close to zero, in fact, the heating curves of temperatures in damaged and undameged zones have a linear trend.
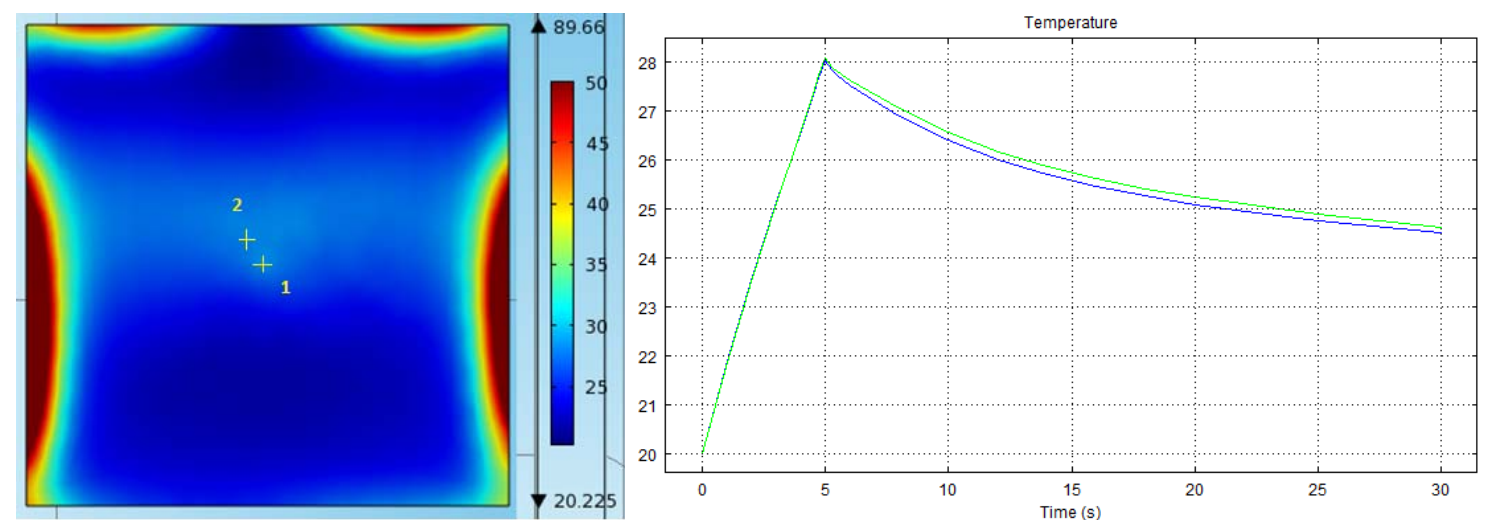

Fig. 18. Defect $1 \times 1 \times 0,5[\mathrm{~mm}]$ placed at depth of 1,3 [mm] (left) with heating-cooling temperature curves (right) 


\section{Conclusions}

In this work a new thermographic technique has been used to assess the damaged zones in composite material by means to a microwaves source. The tests have been carried out on previous damaged composite materials using the innovative technique, lock-in thermographic technique and x-ray. A modified microwaves oven has been used to heating the specimens in order to acquire thermal data by thermocamera.

In the first part of this work a preliminary tests have been carried out in order to determine the best set up in term of best heating time and specimen position in the microwave oven. An innovative algorithm for the data analysis has been developed to evaluate in automatic mode the damaged areas and the various defects typology.

The results obtained with microwaves thermography have been compared with others non destructive techniques such as lock-in thermography and x-ray. Quantitative analysis of the damaged areas show a good agreement among results obtained with microwaves thermography and $\mathrm{x}$-ray while lock-in thermography provides an overestimation of the defects dimensions.

In the second part of the work a numerical analysis have been carried out to support the experimental analysis in terms of chose of the test parameters. As in experimental analysis, the defects are characterized by an high thermal contrast and by a non-linear trend of the heating-cooling temperature curves in damaged and undamaged areas. A good agreement was obtained among the numerical and the experimental analysis.

The thermal and electrical parameters of material were obtained comparing the heating-cooling temperature trend of numerical and experimental analysis.

The numerical analysis can provides the sensibility of the microwave thermography technique. The size of the minimum resolvable defect was $2 \times 2 \times 0,5[\mathrm{~mm}]$, with a depth of $1,3[\mathrm{~mm}]$ with a thermal contrast of [2 ${ }^{\circ} \mathrm{C}$. Further simulations were carried out to verify the sensitivity of the model to verify the detection of smaller sizes defects.

The microwaves thermography technique provides advantages in term of time of tests and the possibility to detect defects at high depth. Future works will focus on experimental setup, in particular will be evaluated the use of microwave antenna in order to obtain a uniform microwave beam to avoid non uniform heating of analyzed specimens.

\section{REFERENCES}

[1] Wu D., Busse G., "Lock-in thermography for non-destructive evaluation of materials". Rev. Gen. Therm., vol. 37, pp. 693-703, 1998.

[2] Meola C., Carlomagno G.M., Giorleo L. The use of infrared thermography for materials characterization. Journal of materials processing Technology, 2004, 155-156, 1132-1137.

[3] Meola C., Carlomagno G.M., Squillace A., Vitello A., "Non-destructive evaluation of aerospace materials with lock-in thermography". Engineering Failure Analysis, vol. 13, pp. 380-388, 2006.

[4] Giorleo G., Meola C., "Comparison between pulsed and modulated thermography in glass-epoxy laminates". NDT\&E International, vol. 35, pp. 287-292, 2002.

[5] Sakagami T., Kubo S. Development of a new non-destructive testing technique for quantitative evaluations of delamination defects in concrete structures based on phase delay measurement using lock-in thermography. Infrared Physics \& Technology, 2002, 43, 311-316.

[6] Stanley, P., Chan, W.K. Quantitative stress analysis by means of the thermoelastic effect. Journal of Strain Analysis, 1985, vol. 20,129-137.

[7] Galietti U., Pappalettere C. Analisi del danneggiamento su componenti in materiale composito con tecnica termoelastica. AIAS XXXVIII Convegno Nazionale, 2009.

[8] Swiderski W., Szabra D., Wojcik J., "Nondestructive evaluation of aircraft components by thermography using different heat sources". Proceedings of 6th Quantitative InfraRed Thermography conference, paper QIRT200679 Dubrovnik (Canada), 2006.

[9] T. Santos, L. C. Costa, M. Valente, J. Monteiro, J. Sousa, "3D Electromagnetic Field Simulation in Microwave Ovens: a Tool to Control Thermal Runaway". Proceedings of the COMSOL Conference, Paris (France), 2010.

[10] COMSOL Multiphysics user guide.

[11] Renato Giannoccaro, PhD Thesis, Design and Testing of aeronautical structures innovative materials 2008. 